\title{
Correction to: The phosphorylation of CD147 by Fyn plays a critical role for melanoma cells growth and metastasis
}

Xu Zhang • Zunnan Huang $\mathbb{D} \cdot$ Yeye Guo - Ta Xiao • Ling Tang · Shuang Zhao • Lisha Wu • Juan Su • Weiqi Zeng • Hongbin Huang $\cdot$ Zheng $\mathrm{Li} \cdot$ Juan Tao $\cdot$ Jianda Zhou $\cdot$ Xiang Chen $\mathbb{D} \cdot$ Cong Peng

Published online: 22 February 2021

(c) The Author(s), under exclusive licence to Springer Nature Limited 2021

Correction to: Oncogene

https://doi.org/10.1038/s41388-020-1287-3

C

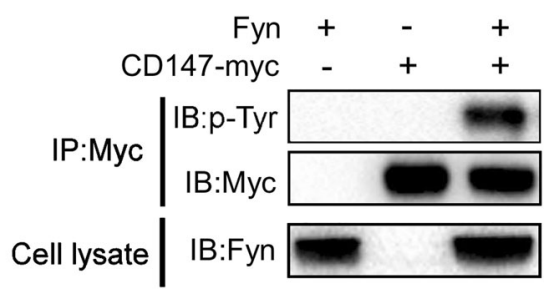

Unfortunately, of the original published version an error occured in Fig. 1c. The correct Figure is given below.

The original version was corrected.

Fig. 1 